\title{
Nonlinear bound-free pair creation in the strong electromagnetic fields of a heavy nucleus and an intense x-ray laser
}

\author{
C. Müller ${ }^{1}$, A. B. Voitkiv² , and N. Grün ${ }^{1}$ \\ ${ }^{1}$ Institute for Theoretical Physics, University of Giessen, \\ Heinrich-Buff-Ring 16, D-35392 Giessen, Germany \\ ${ }^{2}$ Max-Planck-Institut für Kernphysik, \\ Saupfercheckweg 1, D-69117 Heidelberg, Germany
}

\begin{abstract}
We study $e^{-} e^{+}$pair creation in the collision of a heavy nucleus with an intense $\mathrm{x}$-ray laser, where the produced $e^{-}$is simultaneously captured into the K-shell of the projectile nucleus. The parameters of the colliding system are chosen such that the absorption of at least two photons from the laser wave is required in order to surmount the energy threshold of the reaction. Considering this fundamental nonlinear strong-field process for the first time, we estimate the total production rate as well as the angular and energetic distributions of the emitted positrons. According to our results, the process of nonlinear bound-free pair creation could become observable by the aid of the upcoming x-ray laser facilities.
\end{abstract}

PACS numbers: 42.50.Hz, 25.75.Dw, 32.80.-t 
In the early years after the construction of the first laser in 1960, several theoretical physicists addressed the question, whether $e^{-} e^{+}$pair creation in the strong field of a coherent electromagnetic wave is possible [1]. However, the laser intensities and frequencies, that were found to be required for the experimental observation of such a process, were far beyond reach at that time. At present, the interest in this fundamental question has been revived by the remarkable progress in laser technology over the last two decades. In particular, the x-ray free-electron laser (XFEL) facilities that are presently being built at SLAC and DESY [2] seem to provide a promising tool for the experimental study of laser-induced pair creation [3]. These new x-ray sources are proposed to yield spatially coherent synchrotron radiation with single photon energies up to $\hbar \omega \sim 10 \mathrm{keV}$ at intensities close to $I \sim 10^{20}$ $\mathrm{W} / \mathrm{cm}^{2}$. This corresponds to an electric field strength of $F \sim 10^{11} \mathrm{~V} / \mathrm{cm}$ and to an intensity parameter of $\xi=e F / m \omega c \sim 10^{-3}$. Hence, in contrast to visible or infrared lasers at this intensity, ponderomotive effects are negligibly small in an XFEL. For this reason, energy thresholds of strong-field processes are significantly lower in an XFEL than in low-frequency lasers [4]. In a strong-field environment, due to its large photon number density, nonlinear processes, involving the simultaneous emission and/or absorption of more than one photon, can occur with a non-negligible probability. The rate for an $n$-photon process scales as $\xi^{2 n}$ in the $\xi \ll 1$ regime.

A single laser wave, inasmuch as it is a plane wave, cannot extract pairs from the vacuum, no matter how intense it is [5]. Hence, an additional agent is required in order to create pairs in a plane-wave laser field. Against this background, several authors have recently studied pair creation in the electric field of a standing wave formed by two crossed XFEL beams [3]. Another conceivable possibility for the required additional source of electromagnetic energy is the Coulomb field of a charged particle, e.g., an electron [6] or a nucleus [7]. However, if the particle is at rest, then the absorption of about $10^{2}-10^{3}$ XFEL photons is required in order to exceed the energy threshold. Because of the $\xi^{2 n}$ scaling, the resulting production rate will be negligibly small. To overcome this problem, one can consider pair creation by a nucleus moving at relativistic energy through an intense laser field, i.e., pair creation in a laser-nucleus collision [8]. Then, in the rest frame of the nucleus the photon energy is doppler-shifted, which considerably lowers the required number of laser photons to be absorbed.

When a nucleus collides with a laser beam, not only free [8] but also bound-free pair creation is possible, i.e., the $e^{-}$is created in a bound state of the nucleus. For a single $\gamma$-quantum of sufficiently high energy impinging on a nucleus at rest, this process has, in principle, been considered long ago [9]. But, to the best of our knowledge, pair creation with capture by simultaneous absorption of more than one photon has never been treated so far.

Bound-free pair creation in a laser field can be viewed as a photon-induced transition of a bound $e^{-}$to the negative continuum, reversed in time. Thus, there is a close relation with the process of atomic photoionization. A relativistic $S$-matrix approach to nonlinear photoionization of hydrogen in a strong laser field has been developed in Ref.[10]. In the spirit of the strong-field approximation [10], the amplitude for bound-free pair creation with the $e^{-}$captured into the K-shell can be written as

$$
S_{\mathrm{bf}}=\frac{i e}{\hbar c} \int \bar{\Phi}_{1 \mathrm{~s}} \gamma^{\mu} A_{\mu} \Psi_{q} d^{4} x
$$

taken in the nuclear rest frame. Here, $\Phi_{1 \mathrm{~s}}$ is the Coulomb-Dirac wave function of the hydrogenlike ground state of the projectile nucleus of charge $Z, A^{\mu}$ denotes the classical 
four-potential of a circularly polarized, monochromatic laser field of frequency $\omega$ and wavevector $\mathbf{k}$, and $\Psi_{q}$ is a Volkov-Dirac wave function of the negative continuum satisfying $\left(i \hbar c \gamma^{\mu} \partial_{\mu}+e \gamma^{\mu} A_{\mu}-m c^{2}\right) \Psi_{q}=0$ and describing the motion of the $e^{+}$in the laser wave (see, e.g., Ref. [11]). This motion is characterized by the so-called effective four-momentum $q^{\mu}=\left(E_{q} / c, \mathbf{q}\right)$, which is related to the the ordinary free four-momentum $p^{\mu}$ by

$$
p^{\mu}=q^{\mu}-\xi^{2} \frac{m^{2} c^{4}}{2\left(\omega E_{q}-c^{2} \mathbf{k} \cdot \mathbf{q}\right)} k^{\mu} .
$$

For $\xi \ll 1$, the difference between $q^{\mu}$ and $p^{\mu}$, which is caused by the ponderomotive potential of the laser, generally is very small. The effective four-momentum gives rise to an effective mass $m_{*}$ according to $m_{*}^{2} c^{2}=q_{\mu} q^{\mu}=\left(1+\xi^{2}\right) m^{2} c^{2}$. The approximate amplitude (1) is expected to give a good description of the creation process, if the following two conditions are fulfilled. First, the influence of the nuclear Coulomb potential on the bound $e^{-}$state should be much more important than that of the laser field. This is the case, if the electric field of the laser is small compared to the Coulomb field experienced by the K-shell $e^{-}$, and/or it oscillates with a frequency that is high in comparison with the orbital frequency of the bound $e^{-}$. Second, for the emitted $e^{+}$the situation should be reversed, i.e., here the effect of the Coulomb field is supposed to be small compared to that of the laser field. This requirement is satisfied, e.g., if the relative velocity between $e^{+}$and nucleus is high enough.

In Eq. (1), the perturbation that induces the transition, is the four-potential of the laser field. This potential, which is fully accounted for in the "initial" state $\Psi_{q}$, is not included in the "final" state $\Phi_{1 \mathrm{~s}}$. Therefore, the amplitude (1) is said to be expressed in the post form. We note that the corresponding prior form, where the transition is induced by the nuclear Coulomb potential, leads to the same differential pair creation rate. In the nuclear rest frame, this rate can be expressed as

$$
\frac{d^{2} R_{\mathrm{bf}}}{d E_{q} d \cos \theta_{q}}=\frac{m c^{2}}{\hbar} Z^{5} \sum_{n \geq n_{0}} \xi^{2 n} T_{n} \delta\left(E_{q}+E_{1 \mathrm{~s}}-n \hbar \omega\right)
$$

with $\theta_{q}=L(\mathbf{q}, \mathbf{k})$ and $E_{1 \mathrm{~s}}=m c^{2}\left[1-(\alpha Z)^{2}\right]^{1 / 2}$. In Eq. (3), the sum runs over the number $n$ of absorbed laser photons with $n_{0}$ denoting the smallest possible number required from the law of energy conservation, the latter being ensured by the $\delta$-function. The functions $T_{n}$ entering Eq. (3) mainly depend on the $e^{+}$momentum and the laser frequency. Their analytical expression, however, is rather complicated and, therefore, not given here.

We have applied our theory to the collision of a heavy nucleus of charge $Z=50$, moving at a Lorentz factor of $\gamma=50$, with an intense, circularly polarized XFEL beam of photon energy $\hbar \omega=9 \mathrm{keV}$ and intensity parameter $\xi=7.5 \times 10^{-4}$, which corresponds to an electric field strength of $F=1.75 \times 10^{11} \mathrm{~V} / \mathrm{cm}$ and an intensity of nearly $10^{20} \mathrm{~W} / \mathrm{cm}^{2}$. The collision is assumed to be head-on, i.e., the propagation directions of the collision partners are antiparallel. Then, in the nuclear rest frame, the photon energy as well as the laser field strength are enhanced by the factor $(1+\beta) \gamma$ with $\beta=\left[1-1 / \gamma^{2}\right]^{1 / 2}$ and, thus, amount to $\hbar \omega^{\prime}=900 \mathrm{keV}$ [12] and $F^{\prime}=1.75 \times 10^{13} \mathrm{~V} / \mathrm{cm}$. Here and in the following, we use primed quantities in the nuclear rest frame and unprimed quantities in the lab frame. The ponderomotive energy of a free $e^{-}$in such a laser field amounts to $E_{\text {osc }}=\xi^{2} m c^{2} / 2 \approx 0.14 \mathrm{eV}$. The amplitude and velocity of its quiver motion are given by $r_{\mathrm{osc}}=\xi c / \omega^{\prime} \sim 10^{-14} \mathrm{~cm}$ and $v_{\text {osc }}=\xi c \sim 10^{7} \mathrm{~cm} / \mathrm{s}$, respectively. In comparison with that, the binding energy of an $e^{-}$in the K-shell of the projectile ion is $E_{K}=E_{1 \mathrm{~s}}-m c^{2}=35 \mathrm{keV}$. The K-shell radius amounts to 


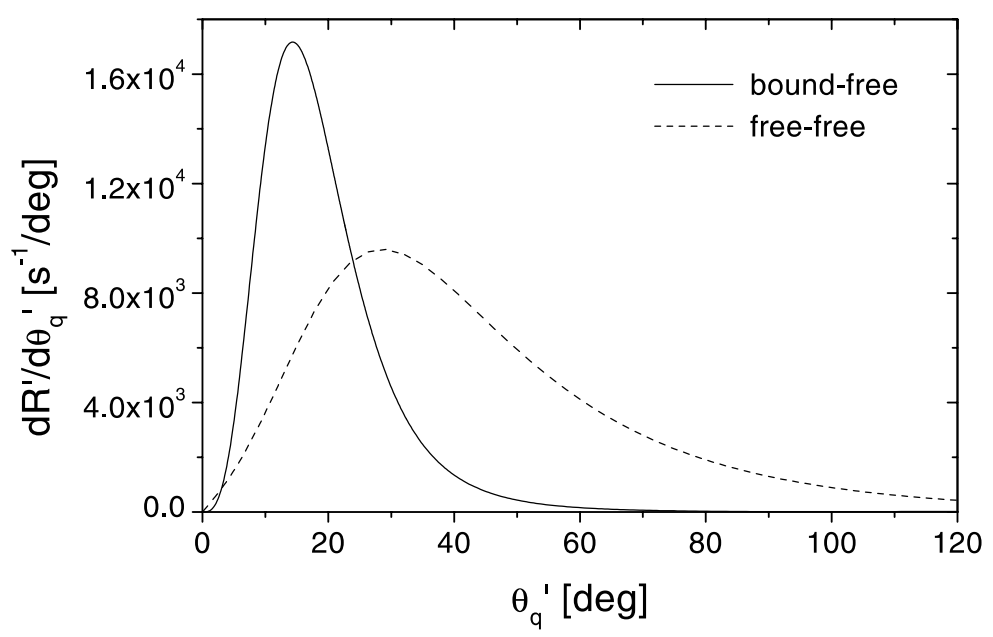

FIG. 1: Nuclear frame rates for nonlinear pair creation, differential in the polar emission angle of the $e^{+}$. The parameters of the colliding system are $Z=50, \gamma=50, \hbar \omega=9 \mathrm{keV}$, and $F=1.75 \times 10^{11}$ $\mathrm{V} / \mathrm{cm}$.

$r_{K} \approx 10^{-10} \mathrm{~cm}$, and the $e^{-}$orbital velocity is $v_{K} \approx \alpha Z c \approx 10^{10} \mathrm{~cm} / \mathrm{s}$. The energy threshold for bound-free pair creation with capture into the K-shell is $\Delta E \approx 2 m c^{2}-E_{K}=987$ $\mathrm{keV}$. This threshold can be surmounted by the absorption of at least two laser photons; the relative contribution of higher photon orders $(n \geq 3)$ is proportional to $\xi^{2}$ and, thus, negligible.

Note that, in the nuclear rest frame, the K-shell Coulomb field of the nucleus, given by $Z e / r_{K}^{2} \approx 6.4 \times 10^{14} \mathrm{~V} / \mathrm{cm}$, is fourty times larger than the laser field strength $F^{\prime}$. In addition, since the laser frequency $\omega^{\prime}$ is much larger than the orbital frequency $\omega_{K}=v_{K} / r_{K}$, the effective distortion of the ground state by the laser field is even weaker than the ratio of the field strengths suggests. Moreover, the values of the binding energy $E_{K}$, K-shell radius $r_{K}$ and orbital velocity $v_{K}$ are orders of magnitude larger than the parameters $E_{\text {osc }}, r_{\text {osc }}$ and $v_{\text {osc }}$ characterizing the oscillatory motion of a free $e^{-}$in the laser wave. Altogether this implies that the influence of the laser field on the bound $e^{-}$is extremely weak. Hence, the Coulomb-Dirac description of the bound $e^{-}$state has clear physical significance. Regarding the state of the created $e^{+}$, we note the following: Since the pair creation occurs practically exclusively by absorption of two laser photons, the $e^{+}$is emitted with a kinetic energy of $2 \hbar \omega^{\prime}-\Delta E \approx 800 \mathrm{keV}$ in the nucleus frame. The corresponding $e^{+}$velocity $v$ gives rise to a Massey parameter of $Z \alpha c / v \approx 0.4$, making the Volkov-Dirac description of the outgoing $e^{+}$ a reasonable approximation.

Based on Eq. (3), we have estimated the total and differential rates for pair creation with capture into the K-shell for the collision system under consideration. Figure 1 shows the angular distribution of the emitted $e^{+}$in the nuclear rest frame, while Figs. 2 and 3 show the energy and angle spectra after Lorentz boost to the lab frame. For comparison, we also give the corresponding rates for nonlinear free-free pair creation [8]. Neglecting the higher photon orders, the positrons originating from bound-free pair creation (the "boundfree positrons", for short) have a fixed total energy of $E_{q}^{\prime}=2 \hbar \omega^{\prime}-E_{1 \mathrm{~s}}=1.32 \mathrm{MeV}$ in the nuclear rest frame. Contrary to that, the energy of the free-free positrons varies continuously between $m_{*} c^{2} \approx m c^{2}$ and $2 \hbar \omega^{\prime}-m_{*} c^{2}=1.29 \mathrm{MeV}$. In both cases, the main part of the 


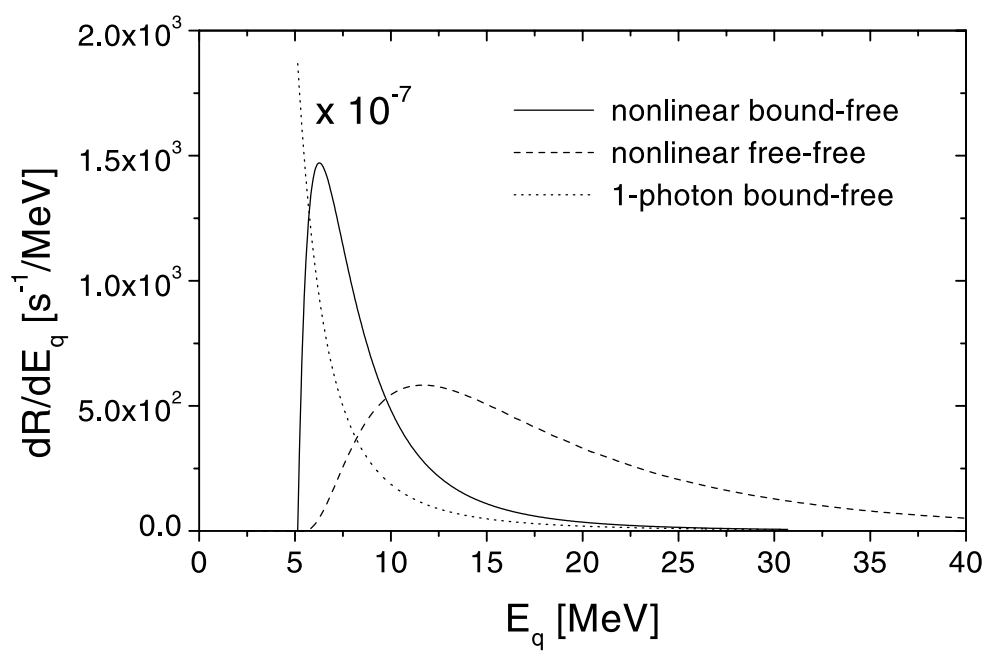

FIG. 2: Lab frame rates for pair creation, differential in the total energy of the emitted $e^{+}$. The collision parameters are the same as in Fig. 1. Also shown is the rate for bound-free pair creation by a single photon of $18 \mathrm{keV}$.

positrons is emitted, roughly speaking, into the propagation direction of the laser beam (see Fig. 1). In the lab frame, as Fig. 2 shows, the energies of the bound-free positrons roughly amount to $5 \mathrm{MeV} \lesssim E_{q} \lesssim 12 \mathrm{MeV}$, which is noticeably smaller than the typical energies of $8 \mathrm{MeV} \lesssim E_{q} \lesssim 30 \mathrm{MeV}$ of the free-free positrons. Integration over $E_{q}$ yields a total rate for nonlinear bound-free pair production of $R_{\mathrm{bf}}=6.5 \times 10^{3} \mathrm{~s}^{-1}$. This means, if the projectile nucleus collides with a laser pulse of $1 \mathrm{fs}$ coherent spike length, then, on average, $3 \times 10^{-12}$ bound-free pairs are created per collision. The total rate for free-free pair production is somewhat larger and amounts to $R_{\mathrm{ff}}=9.6 \times 10^{3} \mathrm{~s}^{-1}$. In addition, Fig. 2 shows the rate for bound-free pair creation by a single photon of twice the energy at the same laser intensity as before. Unlike the nonlinear spectra, the 1-photon rate is a monotonously decreasing function with the maximum value at the smallest possible $e^{+}$energy. (This distinction is connected with the different angular distribution of the 1-photon positrons in the nucleus frame.) The absolute values of the 1-photon rate are orders of magnitude larger because of the weaker intensity dependence.

In contrast to the spectra of Fig. 1, the lab frame angular distributions for nonlinear bound-free and free-free pair creation are qualitatively distinct from one another (see Fig. 3 ). While the free-free positrons smoothly cover the narrow angular range between, say, $177.5^{\circ}$ and $179.8^{\circ}$, practically all positrons from the bound-free channel are emitted into the angle $177.25^{\circ}$, thereby forming an almost discrete spectrum located slightly below the continuous free-free spectrum. The basic reason for the peaky distribution of the bound-free positrons is kinematical by nature: Into the Lorentz transformation from one frame to the other there enters the Jacobian

$$
\left|d \cos \theta_{q}^{\prime} / d \cos \theta_{q}\right| \propto\left|\beta_{+}-\beta \cos \theta_{q}^{\prime}\right|^{-1}
$$

with $\beta_{+}$denoting the reduced velocity of the $e^{+}$in the nuclear rest frame. Since, in this frame, the $e^{+}$has a well-defined energy, the value of $\beta_{+}$is fixed. Apparently, the Jacobian (4) becomes singular at the nuclear frame angle $\theta_{q}^{\prime}=\arccos \left(\beta_{+} / \beta\right)=22.75^{\circ}$, which exactly 


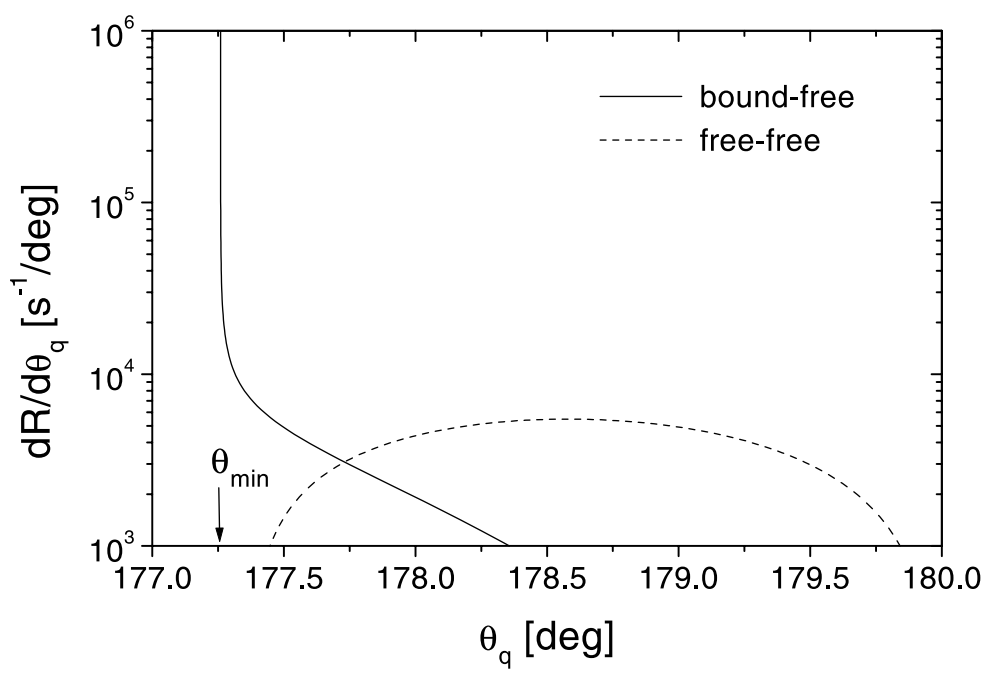

FIG. 3: Same as Fig.1, but given in the lab frame.

corresponds to the lab frame angle $\theta_{q}=177.25^{\circ}$ where the peak in the angular spectrum occurs. Moreover, we note that the latter angle coincides with the minimum lab frame angle that, for kinematical reasons, is accessible to the $e^{+}$. It is given by $\sin \theta_{\min }=-\gamma_{+} \beta_{+} / \gamma \beta$ with $\gamma_{+}=\left(1-\beta_{+}^{2}\right)^{-1 / 2}$ [13]. Hence, essentially all positrons are squeezed into this minimum angle. The above argument does not apply to the free-free positrons, since their energy in the nuclear rest frame is not fixed but varies over a broad range.

Further, we address the question, whether the process of nonlinear bound-free pair creation can be observed in experiment. One possibility would be to aim at the detection of the produced $e^{+}$. According to our results, the total rate for bound-free pair creation is not very large, but since the positrons are emitted with high energy into a definite polar angle, they should be accessible to a measurement, though. Here it is of crucial importance, that the bound-free positrons can be discriminated from the free-free positrons due to their very different spectra. We also notice, that our calculated $e^{+}$spectra, though expressed with respect to the effective momentum, should be directly measureable since $q^{\mu} \approx p^{\mu}$. Alternatively, one could try to detect the hydrogenlike ion formed by the projectile nucleus and the captured $e^{-}$. Certainly, this bound system can decay by the influence of the laser field; but, for the present situation, we estimate the life time of the ground state as $10^{-11} \mathrm{~s}$ in the lab frame [14]. Thus, if we assume the laser to be a fs pulse, most of the bound states will survive and, therefore, also they might serve for the experimental observation of the process.

Finally, we give the scaling behaviour of the total rates for nonlinear bound-free and free-free pair creation, that is, we specify the dependences of $R_{\mathrm{bf}}$ and $R_{\mathrm{ff}}$ on the collision parameters $Z, \gamma, \xi$, and $\omega$. Since in both cases the pair is almost exclusively produced by absorption of two photons, both rates scale as $\xi^{4}$ in the $\xi \ll 1$ regime. Hence, an increase of the laser field strength by one order of magnitude, due to improved x-ray focussing techniques, would lead to a rate enhancement by four orders. Further, $R_{\mathrm{bf}} \propto Z^{5}$ shows the typical $Z$ dependence which is known from bound-free pair production in heavy ion collisions [13], while $R_{\mathrm{ff}}$ scales as $Z^{2}$ due to the first-order treatment of the nuclear Coulomb potential in Ref. [8]. Regarding the remaining parameters $\gamma$ and $\omega$, we note that, in the nuclear frame, the production rates $R^{\prime}$ can only depend on the combination $(1+\beta) \gamma \omega \approx 2 \gamma \omega$, which is 
the doppler-shifted laser frequency $\omega^{\prime}$. Due to time dilation, the relation with the respective lab frame rates is given by $R^{\prime}=\gamma R$. The $\omega^{\prime}$ dependence of the nuclear frame rates $R^{\prime}$ was determined by numerical calculations. As a result, we find the scaling behaviour of the total rates for 2-photon pair creation approximately to be

$$
\begin{aligned}
R_{\mathrm{bf}} & \propto \gamma^{-1} Z^{5} \xi^{4}\left(\omega^{\prime}-\omega_{\mathrm{th}}\right) \\
R_{\mathrm{ff}} & \propto \gamma^{-1} Z^{2} \xi^{4}\left(\omega^{\prime}-\omega_{\mathrm{th}}\right)^{3}
\end{aligned}
$$

with $\omega^{\prime} \approx 2 \gamma \omega$ and $\omega_{\text {th }}$ denoting the respective threshold frequencies for nonlinear pair creation [15].

In conclusion, the fundamental strong-field process of nonlinear bound-free pair creation in the combined electromagnetic fields of a nucleus and a laser has been considered for the first time. We calculated the angular and energetic distributions of the positrons produced in the collision of a relativistic heavy nucleus and an intense XFEL. Interesting peculiarities in the spectra were found, and the scaling behaviour of the total pair creation rates was analyzed. Our results suggest, that nonlinear pair creation could become accessible to experimental observation by the aid of the upcoming XFEL facilities, provided that such a facility is brought together with a suitable ion accelerator. Alternatively, one could replace the ion beam by an $e^{-}$beam in order to study the very closely related process of pair creation with positronium formation in $e^{-}$-XFEL collisions.

[1] N.B. Narozhnyi, A.I. Nikishov, and V.I. Ritus, Sov. Phys. JETP 20, 622 (1965); V.P. Yakovlev, Sov. Phys. JETP 22, 223 (1966); V.S. Popov, Sov. Phys. JETP 34, 709 (1972).

[2] The x-ray free electron laser, TESLA Technical Design Report, edited by G. Materlik and T. Tschentscher (DESY, Hamburg, 2001), Vol. V; J. Arthur, Rev. Sci. Instrum. 73, 1393 (2002).

[3] A. Ringwald, Phys. Lett. B 510, 107 (2001); V.S. Popov, JETP Lett. 74, 133 (2001); R. Alkofer, M.B. Hecht, C.D. Roberts, S.M. Schmidt, and D.V.Vinnik, Phys. Rev. Lett. 87, $193902(2001)$.

[4] W. Becker, Laser Part. Beams 9, 603 (1991).

[5] J. Schwinger, Phys. Rev. 82, 664 (1951).

[6] C. Bamber et al., Phys. Rev. D 60, 092004 (1999).

[7] M.H. Mittleman, Phys. Rev. A 35, 4624 (1987); K. Dietz and M. Pröbsting, J. Phys. B 31, L409 (1998).

[8] C. Müller, A.B. Voitkiv, and N. Grün, Phys. Rev. A 67, 063407 (2003).

[9] E. Fermi and G.E. Uhlenbeck, Phys. Rev. 44, 510 (1933).

[10] H.R. Reiss, J. Opt. Soc. Am. B 7, 574 (1990); Prog. Quant. Electr. 16, 1 (1992).

[11] V.B. Berestetskii, E.M. Lifshitz, and L.P. Pitaevskii, Relativistiv Quantum Theory (Pergamon Press, Oxford, 1971).

[12] Treating the nucleus as a pointlike charge, we disregard the possibilty of photon-induced nuclear excitation.

[13] J. Eichler and W.E. Meyerhof, Relativistic Atomic Collisions (Academic Press, San Diego, 1995). 
[14] The quite rapid decay of the bound system does not conflict with the Coulomb-Dirac description of the $e^{-}$state. In the nuclear frame, its life time amounts to $\sim 10^{-13} \mathrm{~s}$, which is much larger than the orbiting time of $2 \pi / \omega_{K} \sim 10^{-19} \mathrm{~s}$.

[15] We stress that our theoretical approaches are not justified near to the thresholds, since the influence of the nuclear Coulomb field on the resulting low energy $e^{+}$is not small. 